\title{
Producão científica e inserção internacional da revista Scire no período de 2006 a 2014
}

Producción científica e inserción internacional de la revista Scire en el perido 2006-2014

Scientific production and international insertion of the journal Scire in the 2006-2014 period

\author{
Ely Francina Tannuri de OliveIRA (1), Bruno Henrique Alves (2), \\ Marcos Rodrigues do Prado (3), Maria Aparecida PAVANelLi (4)
}

(1) Pesquisadora do Programa de Pós-Graduação em Ciência da Informação da UNESP-Marília-Brasil-Endereço: Rua Coronel José Brás, $n^{\circ} 155$, apartamento, $n^{\circ}$ 502-Marília-SP-Brasil-CEP: 17501-570, etannuri@gmail.com; (2) Doutorando do Programa de Pós-Graduação em Ciência da Informação da UNESP-Marília-Brasil-Endereço: Rua Sargento Wilson Abel Oliveira, $n^{\circ} 1267$ - Garça-SP-Brasil-CEP:17400-000, brhenriqueal-ves@gmail.com; (3) Doutorando do Programa de Pós-Graduação em Ciência da Informação da UNESP-Marília-Brasil-Endereço: Rua Abuassali Abujamra, n 389-Ourinhos-SP-Brasil-CEP: 19907-170, marcospraddo@yahoo.com.br; (4) Doutoranda do Programa de Pós-Graduação em Ciência da Informação da UNESP-Marília-Brasil-Endereço: Avenida Sebas-tião Lacerda Corrêa, nº 928-Araraquara-SP-Brasil-CEP:14800-480, cidinhapavanelli@gmail.com

\section{Resumen}

Se analiza bibliométricamente la producción científica de la revista Scire: Representación y Organización del Conocimiento, para comprobar su internacionalización, las instituciones más productivas en sus respectivos países y las coautorías internacionales y temáticas más relevantes en el periodo 20016-2014. Se evidenciaron 73 instituciones de las cuales 23 publicaron al menos un artículo. Se mapearon las temáticas de trabajo según la clasificación de Dahlberg. Las instituciones fueron agrupadas en clústeres según su similaridad temática y representadas en un dendograma. Se concluye que existe una tendencia, aunque frágil, a su internacionalización, especialmente en relación a los países de América Latina.

Palabras clave: Organización del conocimiento. Producción científica. Revistas científicas. Scire. Coautoría institucional. Sistema de clasificación para la literatura sobre Organización del Conocimiento. América Latina.

\section{Introdução}

Visando à divulgação disseminação e visibilidade dos resultados das pesquisas realizadas, a revista Scire: Representación y Organización del Conocimiento é uma publicação semestral da Universidade de Zaragoza (Espanha), criada em 1995, de caráter interdisciplinar, que trata da representação, normalização, tratamento, recuperação e comunicação da informação e do conhecimento. Em seu editorial, do primeiro número da Revista Scire: Representación y Organización del Conocimiento, em 1995, vol. 1, n.1, Francisco Javier Garcia Marco, seu editor e diretor, explicita as razões do princípio da nova revista: "A revista

\begin{abstract}
A bibliometric analysis of the scientific production of the journal Scire: Representación y organización del Conocimiento in the 2006-2014 period is offered, in order to show its internationalization. Specifically, the study aims to highlight institutions with higher productivity in their countries, as well as to examine institutional co-authorships, highlighting the international coauthorships and the most important themes. As total of 73 institutions were investigated, with 23 publishing at least two investigations. A network of institutional collaboration was created, a list of themes worked by the various collaborating institutions was developed, and the institutions were grouped according to Dahlberg's categories. The institutions and themes were grouped into clusters according to thematic similarity and presented through a dendrogram. As a conclusion, it was observed that the internationalization of the journal Scire is a trend, although fragile, especially in relation to Latin America.
\end{abstract}

Keywords: Knowledge organization. Scientific production. Internationalization. Scire. Institutional co-authorship. Classification System for Knowledge Organization Literature. Latin America.

Scire: Representación y Organización del Conocimiento quer ser mais que um instrumento de difusão. Nossa revista, antes de tudo, é o ponto de encontro de um grupo de pessoas de distintos âmbitos científicos e profissionais preocupados com o mesmo problema: a representação e organização do conhecimento" (Garcia-Marco, 1995).

Seu índice h é 3 e se encontra indexada na base de dados Scopus, desde 2011, além das bases DIALNET, Latindex, Fuete Académica Premier Lisa e Lista. Recebeu o selo de qualidade FECYT e se encontra indexada na plataforma e-revistas (plataforma de acesso aberto de Revistas Científicas Eletrônicas Espanhola e Latino-Americanas). Atualmente é uma revista pertencente ao $3^{\circ}$ 
Quartil, observando-se que, inicialmente quando foi criada, pertencia ao $4^{\circ}$ Quartil, que desde sua indexação na Scopus (2011) mostrou acentuada ascendência. Segundo o Scimago Journal \& Country Rank, dando destaque para os anos de 2012, 2013 e 2014 se produziu 19, 44 e 62 documentos respectivamente, sinalizando assim um crescimento nos últimos três anos (1). Apesar desse visível crescimento, a revista Scire tem, Qualis CAPES B1, no Brasil, provavelmente devido aos diferentes critérios de avaliação utilizados pela Scopus.

A escolha deste periódico deve-se, inicialmente, à relevância do mesmo na área de Ciência da Informação, bem como à carência de estudos realizados nas poucas revistas já consolidadas na temática 'Organização e Representação do Conhecimento', além do aumento significativo de pesquisas na área. Observe-se que, considerando a subárea Biblioteconomia e Ciência da Informação, a Espanha publica 8 revistas, sendo que a revisa Scire: Representación y Organización del Conocimiento se coloca na $4^{a}$ posição entre elas ( $3^{\circ}$ Quartil). A revista Cybermetrics é a única do $1^{\circ}$ Quartil, as revistas Profesional de la Informacion e Revista Española de Documentación Científica no $2^{\circ}$ Quartil e Revista General de Informatión y Documentatión, Anales de Documentatión, lbersid e BiD no $4^{\circ}$ Quartil (Scimago Journal \& Country Rank, 2015).

Na revisão de literatura sobre a análise ou estudos realizados sobre a revista Scire: Representación y Organización del Conocimiento, destacam-se recentes pesquisas em formato de artigo, a saber: "Análise bibliométrica da revista Scire: um estudo de colaboração científica institucional, no período de 1996 a 2010 (Alves, Oliveira e Gracio, 2012) e um segundo estudo denominado "Relações Teóricas da Organização do Conhecimento como com Abordagens de catalogação de Assunto, Indexação e Análise documental: uma Análise de domínio da revista Scire (1995-2010)" (Guimarães, Pinho e Ferreira, 2012). Este último artigo realiza uma análise de domínio da revista Scire (1995-2010), a fim de identificar a produção científica nas linhas teóricas mencionadas, bem como relações existentes entre elas. Ainda, Guimarães et al. (2014) estudam a dimensão temática da pesquisa em OC por meio da análise de domínio dos congressos da ISKO (Brasil, Espanha e América do Norte), com o objetivo de identificar os temas e as características da colaboração científica.

Nos últimos anos, os pesquisadores tem estudado questões relativas a internacionalização do conhecimento científico e de forma mais específica, à revista Scire. Assim, cita-se o trabalho intitulado "International Comparative Domain
Analysis in Knowledge Organization Research Topics in Four Countries - Brazil, South Korea, Spain and the United States" (Beak, Glover, Martínez-Ávila e Milani, 2013).

No sentido de apontar as justificativas para este estudo destaca-se que, com a globalização imposta ao mundo, acirra-se a exigência pela internacionalização da pesquisa e dos periódicos científicos de forma mais instigante. Assim, a grande utilidade dessa pesquisa é avaliar o alcance que os artigos publicados pela revista em questão, são absorvidos por outros paises externos à Espanha. Ainda, considera-se relevante que os autores potenciais que queiram nela publicar, tenham parâmetros substanciais para realizar tal escolha. São estas contribuições que a subárea Estudos Métricos da Informação oferecem, no sentido de avaliação da ciência produzida no país, além do seu impacto, visibilidade e consequente inserção em âmbito local e internacional.

A 'Organização e Representação do Conhecimento (ORC)' foco principal da revista, é a área que trata do desenvolvimento de técnicas para construção, gestão, uso e avaliação de classificações científicas, taxonomias, nomenclatura e linguagens documentais. Trata, ainda, de metodologias de uso e recuperação por linguagem natural, bem como das atividades baseadas nas práticas de armazenagem e recuperação de documentos no decorrer de um processo de aperfeiçoamento da sistematização do conhecimento humano, tendo como objeto de estudo o "conhecimento socializado" (Esteban-Navarro e GarciaMarco, 1995; Barité, 2001; Fujita, 2001).

Segundo Fujita (2008) destacam-se dois aspectos na representação do conhecimento, considerando a área de Ciência da Informação: o resultado da representação do conhecimento por meio da identificação dos conceitos e a representação da estrutura lógica do conhecimento, que resulta na 'Organização do Conhecimento (OC)'. Ainda, segundo Dahlberg (2006), a representação do conhecimento compreende a estrutura lógica da representação conceitual, e, também, o resultado da identificação de conceitos, em função da terminologia utilizada, por meio dos termos utilizados.

A pesquisa em ORC resulta de um contexto dinâmico de produção e reelaboração de conhecimentos. Estes se transformam continuamente em razão da sua aplicação concreta e da comunicação científica, pois as atividades de pesquisa são determinadas pelo contexto social e histórico, pelos marcos teóricos e pressupostos científicos e também pelas diferentes metodologias existentes. 
Há diferentes procedimentos na pesquisa em $\mathrm{ORC}$, tanto relativo à construção do conhecimento, como em relação ao contexto da produção, entre eles as avaliações da produção científica por meio dos estudos bibliométricos. Estes, associados às análises contextuais, evidenciam o referencial teórico-epistemológico dominante na área, as relações existentes, constituindo um dos instrumentos metodológicos que contribuem para a visualização do comportamento da ciência produzida em uma dada área. Gómez-Caridad et al. (2004) observa que dentro de produção científica destacam-se artigos, resumos de congressos, carta, revisão, material editorial, notícias de artigos, biografias, debates, entre outros documentos.

Os indicadores básicos de produção são constituídos pela contagem do número de publicações dos pesquisadores, instituições ou país, e objetivam refletir o impacto junto à comunidade científica à qual pertencem, dando visibilidade àqueles pesquisadores, instituições ou países mais produtivos, bem como às temáticas mais destacadas de uma área do conhecimento. A relevância de se conhecer as temáticas mais destacadas de determinada área do conhecimento, é o fato das temáticas mais estudadas sinalizar as questões em torno das quais os estudiosos desenvolvem suas pesquisas, além de se constituírem focos de interesse daquela área do conhecimento e traduzirem, muitas vezes, os interesses econômicos, sociais e políticos do contexto local.

A colaboração científica entre autores, instituições ou países supõe uma consociação de hipóteses e objetivos centrais de um projeto, o estabelecimento de uma divisão de trabalho, a interação entre os investigadores e o compartilhamento de informação e conhecimento (Olmeda Gómez, Perianes-Rodriguez e Ovalle-Perandones, 2008). Katz e Martin (1997) apontam a coautoria como indicador da atividade de colaboração científica, que possibilita identificar a cooperação nacional ou internacional, bem como descrever a estrutura social e científica de um grupo, que pode ser representada por uma rede social. Neste contexto, a análise de coautoria reflete um rol possível de intercâmbios e trocas, sendo medida pelo número de publicações em colaboração entre autores, instituições ou países.

Considerando o exposto, esta pesquisa objetiva analisar, por meio de procedimentos bibliométricos, a produção científica da revista Scire, a fim de evidenciar a produção científica e inserção internacional da mesma no período de 2006 a 2014. De forma mais específica, objetiva-se destacar as instituições mais produtivas e seus respectivos países de origem, bem como analisar as coautorias institucionais e explicitar as temáticas que mais têm contribuído para os debates e desenvolvimento do saber no tema, no período estudado, temas estes classificados segundo as categorias de Dahlberg (1993). E ainda, estabelecer a relação instituições e temáticas por meio de representações gráficas e analíticas.

\section{Metodologia}

Para levantamento de dados, tomaram-se os 18 volumes da revista Scire, de 2006 até 2014, que constam no endereço eletrônico: http://www.ibersid.eu/ojs/index.php/scire/issue/archive e, manualmente constituiu-se o rol do conjunto de 154 artigos publicados no período. Inicialmente, levantaram-se as 73 instituições que mais se fizeram visíveis na revista, bem como os países a que estas pertenciam com a finalidade de se destacar os países paticipantes da internacionalização. A Tabela I (na página seguinte) apresenta o rol das 23 instituições que produziram pelo menos 2 artigos no período de 2006 a 2014.

Considerando o objetivo de se analisar as coautorias institucionais, levantou-se o total de 41 instituições que fizeram coautorias e a partir delas, gerou-se a rede de colaboração institucional, por meio do software Pajek, relativa a todo período em estudo. Utilizou-se o indicador de densidade, que permite avaliar a coesão e a estrutura da rede. Em relação aos indicadores de centralidade, calculou-se a centralidade de grau e a centralidade de intermediação, por serem os indicadores com maior significação no caso de coautorias. A primeira avalia o número de ligações que uma instituição tem, com as demais da rede (Otte e Rousseau, 2002), mostrando assim aquelas que mais fizeram parcerias. A centralidade de intermediação pode ser definida como o número de vezes que um nó (instituição) necessita para alcançar um outro nó, isto é, o número de caminhos mais curtos, que passam por determinado nó, colocando em evidência as instituições mais importantes da rede. Avaliou-se as instituições de maior inserção internacional que publicaram na revista Scire, no período em estudo, bem como as tendências de ampliação das subredes formadas.

Para explicitar as temáticas que mais têm contribuído para os debates e desenvolvimento do saber no tema, somente foram utilizados os artigos cujos autores trabalharam em coautoria, em um total de 83 artigos ( $54 \%)$, do total de 154 artigos. Estas foram sistematizadas segundo o instrumento de classificação temática das categorias de Dahlberg (1993), o Classification Scheme for Knowlegde Organization Literature (CSKOL). Para a classificação utilizou-se o título do artigo, resumo e as palavras-chave. Em alguns casos foi 
necessário a análise do artigo na íntegra. Uitlizou-se o cluster via apresentação do dendograma, utilizando-se o método Ward com distância euclidiana, sendo as instituições agrupadas por similaridade temática.

\section{Apresentação e análise dos resultados}

Em relação ao total de 73 instituições, encontraram-se 23 instituições mais produtivas, com pelo menos 2 artigos publicados. Assim, do total de instituições, 50 (68,5\%) foram autoras de um único artigo no período. Apresenta-se a Tabela I com o rol das 23 instituições que produziram pelo menos 2 artigos no período de 2006 a 2014.

Destacando as origens das 23 instituições, registra-se que 13 delas são da Espanha (56,5\%), 7 do Brasil, uma do México, uma do Reino Unido e uma do Uruguai. Assim, 39\% das publicações da revista Scire são originárias da América Latina, valor representativo, porém há baixa inserção em outros continentes, justificado pela indexação, em 2012, portanto recentemente da revista em bases internacionais.

\begin{tabular}{|c|c|c|c|c|c|c|c|c|c|c|}
\hline Instituições & 2006 & 2007 & 2008 & 2009 & 2010 & 2011 & 2012 & 2013 & 2014 & Total \\
\hline Universidade Estadual Paulista-FFC-Marília (Brasil) & 3 & 1 & 0 & 2 & 6 & 2 & 6 & 3 & 5 & 28 \\
\hline Universidade de Salamanca (Espanha) & 5 & 0 & 3 & 0 & 1 & 2 & 3 & 1 & 0 & 15 \\
\hline Universidade de Murcia (Espanha) & 1 & 1 & 2 & 1 & 2 & 1 & 0 & 1 & 2 & 11 \\
\hline Universidade de Extremadura (Espanha) & 1 & 4 & 1 & 0 & 1 & 0 & 1 & 0 & 0 & 8 \\
\hline Universidade Rey Juan Carlos (Espanha) & 1 & 3 & 0 & 0 & 2 & 0 & 0 & 0 & 0 & 6 \\
\hline Universidade Carlos III de Madrid (Espanha) & 1 & 0 & 0 & 2 & 0 & 2 & 0 & 1 & 0 & 6 \\
\hline Universidade Complutense de Madrid (Espanha) & 1 & 0 & 2 & 0 & 0 & 0 & 1 & 1 & 0 & 5 \\
\hline Universidade de Zaragoza (Espanha) & 0 & 0 & 0 & 1 & 0 & 1 & 0 & 2 & 1 & 5 \\
\hline Universidade de São Paulo (Brasil) & 1 & 1 & 0 & 0 & 0 & 0 & 0 & 1 & 1 & 4 \\
\hline Universidade de Granada (Espanha) & 0 & 1 & 1 & 0 & 1 & 1 & 0 & 0 & 0 & 4 \\
\hline Universidade Nacional Autónoma de México & 0 & 3 & 1 & 0 & 0 & 0 & 0 & 0 & 0 & 4 \\
\hline Universidade Federal de São Carlos (Brasil) & 0 & 0 & 0 & 0 & 0 & 1 & 1 & 1 & 0 & 3 \\
\hline Universidade de Alcalá de Henares (Espanha) & 1 & 0 & 0 & 1 & 0 & 0 & 0 & 0 & 1 & 3 \\
\hline Universidade Federal de Santa Catarina (Brasil) & 3 & 0 & 0 & 0 & 0 & 0 & 0 & 0 & 0 & 3 \\
\hline Universidade de Valladolid (Espanha) & 0 & 0 & 0 & 1 & 0 & 0 & 2 & 0 & 0 & 3 \\
\hline Universidade de León (Espanha) & 0 & 0 & 0 & 1 & 1 & 0 & 1 & 0 & 0 & 3 \\
\hline Instituto Brasileiro de Inf. e Tecnológia (Brasil) & 1 & 1 & 0 & 0 & 0 & 1 & 0 & 0 & 0 & 3 \\
\hline Universidade Autónoma de Barcelona (Espanha) & 0 & 0 & 0 & 0 & 0 & 2 & 1 & 0 & 0 & 3 \\
\hline Universidade Federal Fluminense (Brasil) & 1 & 0 & 0 & 0 & 0 & 0 & 0 & 0 & 1 & 2 \\
\hline Universidade Federal de Pernambuco (Brasil) & 0 & 0 & 0 & 0 & 0 & 0 & 1 & 0 & 1 & 2 \\
\hline Universidade de la República (Uruguai) & 0 & 0 & 0 & 1 & 0 & 0 & 0 & 0 & 1 & 2 \\
\hline Cura Consortium and TFPL Ltd (Reino Unido) & 1 & 0 & 1 & 0 & 0 & 0 & 0 & 0 & 0 & 2 \\
\hline Cindoc-Csic (Espanha) & 1 & 0 & 0 & 0 & 1 & 0 & 0 & 0 & 0 & 2 \\
\hline Total & 22 & 15 & 11 & 10 & 15 & 13 & 17 & 11 & 13 & 127 \\
\hline
\end{tabular}

Tabela I. Instituições e países que produziram pelo menos 2 artigos

Portanto, a partir da análise dos dados, o Brasil é o país com maior destaque e inserção na revista em questão. É relevante destacar a estreita relação, quase que histórica entre Espanha e Brasil. Fazendo uma restropectiva, considera-se o Tratado Geral de Cooperação e Amizade entre a República Federativa do Brasil e o Reino da Espanha, em seu $12^{\circ}$ artigo deixa explicitado a necessidade de facilitar o intercâmbio acadêmico entre representantes das repectivas universidades, instituições de pesquisa, educação superior e cultura, bibliotecas, arquivos e outras entidades. Para esse fim, ambas as partes, Brasil e Espanha, acordam estabelecer um sistema de concessão de bolsas de estudo visando a comunicação entre os diferentes países.

Cite-se ainda, que em março de 2001, firma-se o convênio de Cooperação entre o Ministério de 
Educación, Cultura y Deporte (MECD) da Espanha e o Ministério da Educação do Brasil, para o desenvolvimento de programas de formação, aperfeiçoamento e atualização de conhecimentos para pós-graduados e professores universitários (Capes, 2001). Há outros convênios de comparação entre estes países, que fugiriam ao escopo dessa pesquisa. Justifica-se a destacada presença dos pesquisadores brasileiros, em periódicos espanhóis. Cite-se como exemplo, especialmente na Universidade Estadual PaulistaFFC-Marília a presença de Victor Herrero-Solana, da Universidade de Granada, e com continuidade em 2005, com a visita do prof. Felix de
Moya Anégon da mesma universidade, culminando com a vinda do professor visitante Antonio Pulgarín Guerrero-Espanha, da Universidade de Extremadura, em 2010, com auxílio do Conselho Nacional de Desenvolvimento Científico e Tecnológico-CNPq.

A Tabela I apresenta ainda, a produção de artigos por ano, nas diferentes instituições e países. Destaca-se assim, na linha dos totais, a produção de 17 artigos, coincidindo com o ano de indexação da revista. Depois de 2012, a revista volta a ter número de artigos próximo ao que havia antes da indexação.

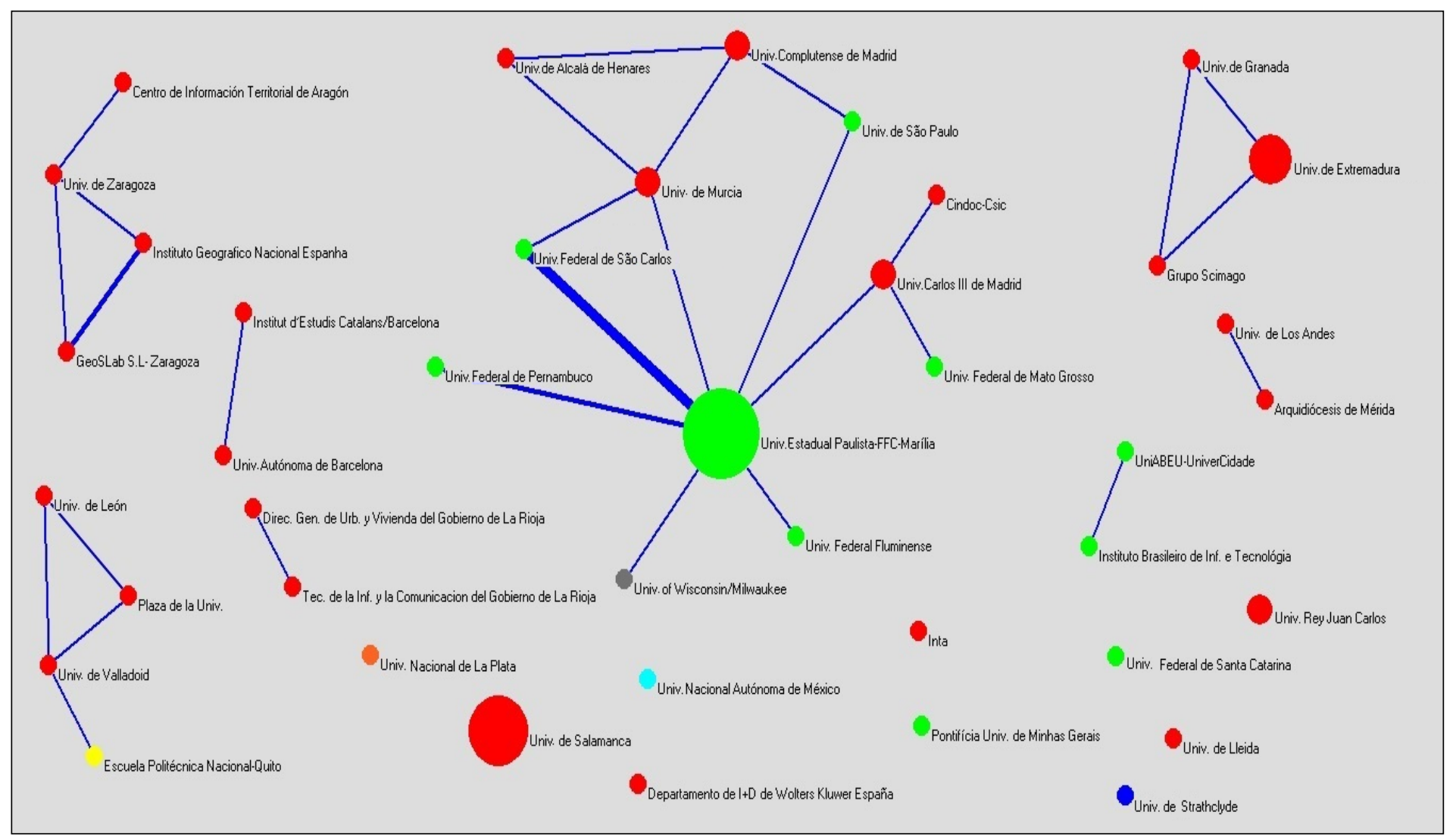

Figura 1. Rede de colaboração institucional (2006 a 2014)

Destaque-se que em 2006, há a maior produção da revista Scire com 22 artigos. Tem-se por hipótese que, em vista da revista ter dedicado um número especial ao tema OC em diferentes fases do ciclo da informação documental, acabou por incentivar e incrementar a pesquisa na referida temática (Garcia-Marco, 2006).

Em síntese, a Tabela I mostra por meio do estudo diacrônico, entre 2006 e 2014, uma produção equilibrada e constante de artigos, com pequenas variações, no momento de sua criação e de sua indexação em base de dados, apesar da referida tabela apresentar somente instituições que publicaram até dois artigos.

Em relação às coautorias, apresenta-se a Figura 1 , com a rede de colaboração institucional construída considerando o período total em estudo, ou seja, todas as coautorias que foram realizadas no período, para que se possa analisar a dinâmica da rede nestes 9 anos.

Os círculos em vermelho são de instituições espanholas, em verde de instituições brasileiras, o azul, amarelo e cinza de outras instituições. Os 
círculos sem ligação alguma, significam instituições, cujos pesquisadores fazem somente coautoria interna; a espessura do segmento significa as frequências de coautorias. Destaca-se a subrede centrada na Universidade Estadual Paulista-FFC-Marília, com o maior número de relações, constituída de sete instituições coautoras. Ainda nesta subrede, ressalta-se o grande número de coautorias institucionais com a Universidade Federal de São Carlos, explicitada pelo segmento mais forte e um pouco mais fraco com a Universidade Federal de Pernambuco, além de parcerias feitas com instituições estrangeiras, como Universidade de Murcia, Universidade Carlos III de Madrid e University of WisconsinMilwaukee e de forma indireta com o CindocCsic, Universidade Complutense de Madrid e Universidade Alcalá de Henares.

Esses dados mostram que a subrede centrada na Universidade Estadual Paulista-FFC-Marília tende à fazer coautorias com outras instituições estrangeiras, consignando o início da internacionalização, sem deixar de fazer suas coautorias com universidades nacionais, tais como a Universidade de São Paulo e Universidade Federal Fluminense.

Observa-se ainda, que a subrede formada pelas instituições espanholas - Universidade de León, Universidade Valadolid, Plaza de la Universidade - adquire características de um grupo internacionalizado, com a coautoria com a Escuela Politécnica Nacional de Quito. Ainda, destacam-se outros grupos espanhóis, a saber: Centro de Información Territorial de Aragón, Universidade de Zaragoza, Instituto Geografico Nacional Espanha e GeoSLab S. L. Zaragoza; Universidade de Granada, Universidade de Extremadura e Grupo SCImago.

Identifica-se na rede de colaboração institucional quatro subredes formadas por duas instituições, sendo três delas espanholas e uma brasileira. Outras instituições espanholas, brasileiras, inglesa, mexicana e argentina, aparecem isoladas somente com coautoria interna. A densidade da rede apresenta-se, aproximadamente, com $4 \%$, justificado pelo número das instituições relativamente alto. A maior centralidade de grau é sete, da Universidade Estadual Paulista-FFC-Marília, caracterizada pelo maior número de ligações, com centralidades menores, tais como, centralidade quatro da Universidade de Murcia e Universidade Carlos III de Madrid e Universidade Complutense de Madrid, com centralidade três. Quanto aos indicadores de intermediação, destacam-se a Universidade Estadual Paulista FFCMarília com 43,50 de intermediação, a Universidade Carlos III de Madrid, com intermediação 19 com intermediação em um total de 7 universidades com valor de intermediação maior que zero, é o número de caminhos mais curtos, que passam por determinado nó (instituição).

Em síntese, a rede de colaboração institucional da revista Scire, nesse período em estudo, apresenta-se constituída por instituições espanholas, com destacada presença de instituições brasileiras, mostrando uma consolidação de parcerias entre Brasil e Espanha, advindas de diferentes convênios institucionais firmados entre estes dois países, que proporcionam pesquisa comuns, intercâmbios de pesquisadores, como também de outras instituições estrangeiras, porém não tão significativas.

Para explicitar as temáticas que mais têm contribuído para os debates e desenvolvimento do saber no tema, no período estudado, temas estes classificados segundo as categorias de Dahlberg, apresenta-se a Tabela II, que se constituiu a partir da construção do dendograma, de forma a agrupar as instituições no entorno de temáticas similares ou próximas.

Buscou-se aporte na concepção filosófica da teoria de Dalhberg (1993), que oferecem fundamentos no sentido de se constituírem em procedimentos instrumentais utilizados nos processos de organização, interpretação e estruturação do conhecimento humano. Para Dahlberg (1993), o Sistema de Classificação da literatura em OC assim se apresenta:

- Categoria 0: Este se refere à divisão de forma dos documentos, na literatura em OC. No entanto, este não é o objeto desta pesquisa, sendo assim, nenhuma pesquisa foi neste grupo classificada.

- Categoria 1: Fundamentos teóricos e problemas gerais da OC, com os seguintes conteúdos: Ordenação e OC, Conceptologia em OC, Matemática e OC, Teoria de sistemas e OC, Psicologia e OC, Ciência e OC, Problemas em OC, Pesquisa da Classificação, História da OC;

- Categoria 2: Sistemas de Classificação e Tesauros, estrutura e construção. Apresentamse os seguintes conteúdos: Estruturas e elementos de Sistemas de Classificação e Tesauros, Construção de Sistema de Classificação, Relações entre conceitos, Taxonomias, Notações e códigos, Manutenção, atualização e armazenagem em C\&T, Concordância entre linguagens de indexação, Avaliação de Sistemas de Classificação e Tesauros;

- Categoria 3: Metodologia de Classificação e Indexação, subentendendo os seguintes conteúdos: Teoria da Classificação e Indexação, 
Análise de Assunto, Técnicas de Classificação e Indexação, Classificação e Indexação Automática, Ordenação Manual e Automática,
Geração de Índices e Programas, Reclassificação, Avaliação de Classificação e Indexação, Codificação e decodificação.

\begin{tabular}{|c|c|c|c|c|c|c|c|c|c|}
\hline Gr. & Instituições & Total & C1 & C2 & C3 & C4 & $C 7$ & C8 & C9 \\
\hline \multirow[t]{10}{*}{ G1 } & Grupo Scimago & 1 & & & & & 1 & & \\
\hline & Univ. Federal de Mato Grosso & 1 & & & & & 1 & & \\
\hline & Univ. de Lleida & 1 & & & & & 1 & & \\
\hline & Escuela Politécnica Nacional-Quito & 1 & & & & & 1 & & \\
\hline & GeoSLab S.L. - Zaragoza & 1 & & & & & 1 & & \\
\hline & Institut d' Estudis Catalans/Barcelona & 1 & & & & & 1 & & \\
\hline & Instituto Geografico Nacional Espanha & 1 & & & & & 1 & & \\
\hline & Tec. de la Inf. y la Com. Del Gov. de La Rioja & 1 & & & & & 1 & & \\
\hline & Univ. Autónoma de Barcelona & 1 & & & & & 1 & & \\
\hline & Direc. Gen. de Urb. y Viv. del Gob. de La Rioja & 1 & & & & & 1 & & \\
\hline \multirow[t]{6}{*}{ G2 } & Univ. de Léon & 2 & & 1 & & & 1 & & \\
\hline & Plaza de la Univ. & 1 & & 1 & & & 1 & & \\
\hline & Univ. de Valladoid & 3 & & 2 & & & 3 & & \\
\hline & Univ. Rey Juan Carlos & 1 & & & & & 2 & & \\
\hline & Dep. I+D de Wolters Kluwer España & 1 & & 1 & & & 1 & & \\
\hline & Univ. de Zaragoza & 2 & & & & & 2 & & \\
\hline \multirow[t]{9}{*}{ G3 } & Instituto Nacional de Técnica Aeroespacial & 1 & & & & & 1 & 1 & \\
\hline & Pontifícia Univ. de Minas Gerais & 1 & 1 & & & & & & \\
\hline & Univ. de Alcalá de Henares & 2 & 1 & & 1 & & 1 & 1 & \\
\hline & Univ. de São Paulo & 4 & 1 & 1 & & & 1 & 1 & 1 \\
\hline & Univ. Federal de Santa Catarina & 2 & 1 & & & & 1 & 1 & 1 \\
\hline & Univ. Federal Fluminense & 2 & 1 & & & & 1 & & \\
\hline & Univ. Nacional de La Plata & 1 & & 1 & & & & 1 & \\
\hline & Univ. of Wisconsin/Milwaukee & 1 & 1 & & & & & & \\
\hline & Univ. Federal de Pernambuco & 2 & 2 & & & & 1 & & \\
\hline \multirow[t]{8}{*}{ G4 } & Univ. de Granada & 3 & & & & & 2 & & 2 \\
\hline & Univ. Nacional Autónoma de México & 2 & & & & & & & 2 \\
\hline & UniABEU-UniverCidade & 1 & & & & & 1 & & 1 \\
\hline & Instituto Brasileiro de Inf. e Tecnológia & 2 & & & & & 1 & & 1 \\
\hline & Univ. de Los Andes & 1 & & & & & & & 1 \\
\hline & Arquidiócesis de Mérida & 1 & & & & & & & 1 \\
\hline & Univ. of Strathclyde & 1 & & & & 1 & & & 1 \\
\hline & Univ. de Aragon & 1 & & & & & & & 1 \\
\hline \multirow[t]{3}{*}{ G5 } & Cindoc-Csic & 3 & & & & & 3 & 2 & \\
\hline & Univ. Carlos III de Madrid & 5 & 1 & & & & 4 & 1 & 1 \\
\hline & Univ. Complutense de Madrid & 4 & & & & & 3 & 1 & 1 \\
\hline \multirow[t]{3}{*}{ G6 } & Univ. de Extremadura & 6 & & & 1 & 1 & 2 & 1 & 3 \\
\hline & Univ. Federal de São Carlos & 3 & 1 & & 3 & & 1 & & \\
\hline & Univ. de Murcia & 6 & 2 & & 2 & & 3 & 2 & 2 \\
\hline G7 & Univ. Estadual Paulista-FFC-Marília & 20 & 10 & 2 & 4 & & 10 & 2 & \\
\hline G8 & Univ. de Salamanca & 15 & & & & & 14 & 1 & 2 \\
\hline
\end{tabular}

Tabela II. Clusters das instituições articuladas com as categorias temáticas de Dahlbeg 
- Categoria 4: Sistemas de Classificação e Tesauros, com os seguintes conteúdos: Dewey Decimal Classification, Library of Congress Classification, Library of Congress Subject Headings, Colon Classification; este Grupo é mais específico do que o Grupo 2.

- Categoria 5: Classificação orientada à objetos (Taxonomias), nas diferentes áreas do conhecimento.

- Categoria 6: Classificações e Tesauros de assuntos específicos.

- Categoria 7: Representação do Conhecimento por Linguagens e Terminologia, com os seguintes conteúdos: Problemas gerais de linguagem natural em relação à $\mathrm{OC}$, Processamento automático da linguagem, Sistemas de recuperação on-line e tecnologias, Problemas de terminologia, entre outros.

- Categoria 8: Indexação e Classificação aplicadas, compreendendo os seguintes conteúdos: Problemas gerais, Catálogos, Diretrizes, Regras, Índices, Indexação e Classificação de dados, títulos, literatura primária, de livros, entre outros.

- Categoria 9: Ambiente da OC, com os seguintes conteúdos: Organização profissional e espacial (nível nacional e internacional) bem como ítens de Educação e treinamento, Legislação, Econômicos, Uso de serviços, Normalização do trabalho de OC, entre outros.

Apresenta-se a Tabela II, com as diferentes instituições formando clusters, segundo as temáticas comuns ou similares.

Para análise dos clusters, destaca-se inicialmente que não foram encontrados artigos nas categorias (C) - C5 e C6, sugerindo assim que a revista Scire não possui como foco de pesquisa as temáticas 'Taxonomias, nas diferentes áreas do conhecimento' ou 'Classificações e Tesauros para assuntos específicos', considerando-se que as diferentes categorias de Dahlberg, não foram mutuamente exclusivas, a soma de cada linha não resulta o total registrado, devido ao fato de um artigo pertencer a uma ou mais categorias.

Encontraram-se oito agrupamentos. O primeiro deles denominado grupo $\mathrm{G} 1$, o maior grupo constituído de 10 instituições, apresentou cada uma delas um único trabalho em coautoria, na $\mathrm{C} 7$, denominada "Representação do conhecimento por linguagens e Terminologia", na qual aborda aspectos do processamento automático da linguagem, terminologia e recuperação da informação. Este grupo tem como foco de investigação os temas em $\mathrm{C} 7$, mas não significativa, devido à sua baixa representatividade na frequência.
O segundo grupo G2, trata das categorias $C 2$ "Sistemas de Classificação e Tesauros, estrutura e construção" e C7 "Representação do Conhecimento por Linguagens e Terminologia". Das seis instituições todas apresentaram artigos em C7 e quatro delas em C2. Destaque-se que essas duas categorias se relacionam por apresentarem sub-temáticas similares, tais como: Relações entre conceitos, Problemas de terminologia, trabaIho de terminologia orientada ao assunto, concordância entre linguagens de indexação, processamento automático da linguagem, entre outros, ampliando assim seu foco de pesquisa.

Constituído por nove universidades, o grupo G3, concentra pesquisas especialmente em $\mathrm{C} 1 \mathrm{de}$ nominado "Fundamentos teóricos e problemas gerais da OC", C7 "Representação do Conhecimento por Linguagens e Terminologia" e C8 "Indexação e Classificação aplicadas", com pouca presença nas demais categorias. Estas instituições se preocupam com os fundamentos teóricos e conceituais, bem como os aspectos aplicados da OC, dispersando seus focos de pesquisas e por outro lado concentrando em C1, C7 e C8.

Todas as oito instituições do grupo G4, apresentam pesquisas em C9 "Ambiente da OC, com menor presença em C7 "Representação do Conhecimento por Linguagens e Terminologia" e C4 "Sistemas de Classificação e Tesauros". O C9 focaliza, especialmente, em organização profissional, bem como itens de educação e treinamento, legislação, estudos de usuários, entre outros.

O grupo G5, formado por três instituições todas elas espanholas, concentra suas pesquisas em C7 "Representação do Conhecimento por Linguagens e Terminologia" e C8 "Indexação e Classificação aplicadas", portanto para os aspectos mais técnicos da Ciência da Informação e de forma mais dispersa em C1 e C9.

As três instituições do grupo G6 contemplam pesquisas em todas as categorias de Dahlberg aqui presentes, exceto em C2, com maior ênfase em C3 e C7, que trabalham metodologias de classificação e indexação.

Ainda, o grupo G7 formado por uma única universidade, a Univerisidade Estadual Paulista-FFCMarília, a mais produtiva, com destaque para $\mathrm{C} 1$ e $\mathrm{C} 7$, categorias que se articulam na medida que tratam de Fundamentos teóricos e questões de OC.

Por fim, o grupo G8 formado pela Universidade de Salamanca com prevalência para pesquisas em $\mathrm{C}$, com frequência 14 nesta categoria e ainda pesquisas em C8 e C9. 
Comparando os dados do cluster, com a rede de colaboração institucional (Figura 1) e com as instituições mais produtivas (Tabela I), destacam-se nas três representações a Universidade Estadual Paulista-FFC-Marília, que no cluster, por ser a instituição mais produtiva aparece em destaque e sozinha; na rede de colaboração institucional, faz coautorias com países, como a Espanha, Estados Unidos da América-EUA e instituições do Brasil, com destaque para a Universidade de São Paulo e a Universidade Federal de São Carlos com maior frequência e centra a maior sub-rede. Analisando a rede de colaboração esta instituição é a que mais possui coautores, dentro do Brasil e com instituições espanholas.

Em relação à Universidade de Salamanca, comparando os dados do cluster, com a rede de coautoria (Figura 1) e com as instituições mais produtivas, destaca-se nas três representações a Universidade de Salamanca, que no cluster e na rede, aparece em destaque e sozinha, por ser a instituição mais produtiva, aparecendo na Tabela I com 15 artigos e na segunda posição, logo após a Universidade Estadual Paulista-FFC-Marília. Não faz interlocução com outras instituições, porém possui grande número de coautorias interna.

\section{Considerações finais}

Este estudo procurou caracterizar os principais momentos que determinaram a criação da revista Scire, desde o editorial do seu primeiro número, o ano em que foi feito um número monográfico da mesma, o ano de sua indexação em bases internacionais, bem como sua produção nas diferentes instituições, por meio de uma análise diacrônica. Identificou-se as instituições e respectivos países que publicaram na revista, para avaliar sua inserção internacional, destacando-se sua elite institucional. Por meio das palavraschave, foram explicitadas as temáticas que contribuíram para os debates e desenvolvimento do saber no tema. Apresentou-se a rede de colaboração institucional em destaque no tema e utilizou-se a classificação de Dahlberg, agrupandose, as instituições por similaridade temática, via representação do dendograma.

A formação das redes no período de 2006 a 2014, mostra a tendência, embora frágil, à internacionalização da revista Scire, especialmente, em relação aos países da América Latina. Observou-se uma rede de colaboração institucional com poucas subredes, onde as instituições são originárias de regiões tais como: América Latina, América Central, especialmente, Cuba e México, ainda América do Norte e Europa.
Há uma maior subrede que sugere a integração e expansão, para uma rede com maior consistência internacional com instituições advindas de diferentes países, bem como instituições sem interlocução com as demais. Ainda, pequenas tríades e díades formadas com instituições advindas do mesmo país, mostrando que a interlocução pouco extrapola os limites geográficos dos seus próprios países. A recente indexação da revista em bases de dados internacionais, explica a pouca coautoria internacional, apesar da relevância temática da revista.

Em relação às temáticas publicadas pela revista, elas têm como foco os fundamentos teóricos da área, sem deixar de lado os procedimentos técnicos da ORC.

No entanto, os agrupamentos das instituições, via dendograma, mostra que a revista Scire tem dois focos principais de pesquisa: C7 'Representação do Conhecimento por Linguagens e Terminologia' e C1 'Fundamentos teóricos e problemas gerais da OC', nas quais apresenta 70 e 22 pesquisas respectivamente, neste período em estudo dentro dos 83 artigos em coautoria (2).

A análise dos dados procurou destacar a articulação existente, entre instituições mais produtivas, presença na rede de colaboração institucional e proximidade temática entre as instituições.

Recomenda-se que este estudo possa servir de fonte para futuros trabalhos que estudam questões de internacionalização de periódicos científicos que tratam deste tema nuclear na área de Ciência da Informação.

\section{Agradecimentos}

Agradecemos, especialmente, ao Conselho Nacional de Desenvolvimento Científico e Tecnológico (CNPq-Brasil)

\section{Notas}

(1) O Quartil é um indicador ou medida de uma posição do periódico em relação a totalidade de sua área. Se dividirmos em 4 partes iguais uma lista de revistas ordenadas do maior para o fator de impacto menor, cada uma dessas partes denomina-se Quartil. Revistas com maior Fator de Impacto (FI) será do primeiro Quartil (pertencem ao grupo dos primeiros $25 \%$ ), os Quartis médios são o segundo e o terceiro Quartil é o de menor $\mathrm{FI}$, está nos últimos $25 \%$ do conjunto todo (Barbetta,1994).

(2) Justifica-se a construção do dendograma feito somente a partir das coautorias, para assim melhor articular dendograma e rede entre si. Ratifica-se ainda que, devido as categorias serem mutuamente excludentes, a quantidade de artigos por categoria não corresponde ao total de artigos. 


\section{Referências}

Alves, Bruno Henrique; Oliveira, Ely Francina Tannuri de; Gracio, Maria Cláudia Cabrini (2012). Análise bibliométrica da revista Scire: um estudo de colaboração científica institucional. // Scire. 18:2 (Julho 2012) 43-48.

Barité, Mario (2001). Organización del conocimiento: un nuevo marco teórico-conceptual en Bibliotecología y Documentación. // Carrara, Kester (Org.). Educação, universidade e pesquisa. Marília: Unesp-Marília-Publicações, 2001. 35-50.

Beak, Jihee; Glover, Jeanette; Martínez-Ávila, Daniel; Milani, Suellen Milani (2013). International Comparative Domain Analysis in Knowledge Organization Research Topics in Four Countries - Brazil, South Korea, Spain and the United States. // Proceedings from North American Symposium on Knowledge Organization. 4:1, 30-39. http://journals.lib.washington.edu/index.php/nasko/article/view/14643 (2017-02-19).

Coordenação de Aperfeiçoamento de Pessoal de Nível Superior (2001). Avaliação da pós-graduação. // CAPES.(2001).http://capes.gov.br/acessoainformacao/perguntas-frequentes/avaliacao-da-pos-graduacao (201606-16).

Dahlberg, Ingetraut (1993). Knowledge Organzation: Its Scope and Possibilities. // Knowledge Organization. 20:4, 211-222.

Dahlberg, Ingetraut (2006). Knowledge organization: a new science?. // Knowledge Organization. 33:1, 11-19.

Esteban Navarro, Miguel Angel; García Marco, Francisco Javier (1995). Las "Primeras Jornadas sobre Organización del Conocimiento: Organización del Conocimiento e Información Científica". // Scire. 1:1 (Enero-Junio 1995) 149-157.

Fujita, Mariângela Spotti Lopes (2001). Organização do conhecimento: algumas considerações para o tratamento temático da informação. // Carrara, Kester. (Org.). Educação, universidade e pesquisa. Marília: Unesp-MaríliaPublicações, 2001. 29-34.

Fujita, Mariângela Spotti Lopes (2008). Organização e Representação do Conhecimento no Brasil: análise de aspectos conceituais e da produção científica do ENANCIB no período de 2005 a 2007 . // Tendência da Pesquisa Brasileira em Ciência da Informação. 1:1, 1-32.

García Marco, Francisco Javier (1995). Editorial. // Scire. 1 (Julio-Diciembre 1995) 1-2.
García Marco, Francisco Javier (2006). Editorial. // Scire.1 (Enero-Junio 2006) 1-2.

Gómez Caridad, Isabel; Fernández Muñoz, María Teresa ; Bordons, María ; Morillo Ariza, Fernanda (2004). Proyeto de obtención de indicadores de producción científica y tecnológicas de España. // Centro de Infoemación y Documentación Científica (CINDOC) - Consejo Superior de Investigaciones Científicas (CSIC), Madrid. (2004). http://digital.csic.es/bitstream/10261/12260/1/informe1.pdf (2016-06-16).

Guimarães, José Augusto Chaves; Pinho, Fábio Assis; Ferreira, Gustavo Meletti. (2012). Relações Teóricas da Organização do Conhecimento como com Abordagens de catalogação de Assunto, Indexação e Análise documental: uma análise de domínio da revista Scire (1995-2010). // Scire. 18:2 (Agosto 2012) 31-41.

Guimarães, José Augusto Chaves et al. (2014). A dimensão temática da pesquisa em organização do conhecimento: uma análise de dominio dos congressos nacionais e regionais da ISKO (Brasil, Espanha e América do Norte). // Scire. 20:2 (Jul.- Dic. 2014) 19-25.

Katz, J. Sylvan; Martin, Ben R. (1997). What is research collaboration?. // Research Policy. 26 (January 1995) 1-18.

Olmeda Gómez, Carlos; Perianez-Rodriguez, Antonio; Ovalle-Perandones, María Antonia (2008). Estructura de las redes de colaboración científica entre las universidades españolas. // Ibersid: Revista Internacional de Sistemas de Información y Documentación, Zaragoza, 2, 129-140.

Otte, E.; Rousseau, R. (2002). Social network analysis: a powerful strategy, also for the Information sciences. // Jounal of Information Science. 28:6 (December 2002), 441-453.

SCImago Journal \& Country Rank (2015). Science analysis. // SJR. (2015). http://www.scimagojr.com/ (2016-06-16).

Scopus (2011). What does it cover?. // Elsevier. (2011). https://www.elsevier.com/solutions/scopus (2016-06-16)

Tratado Geral de Cooperação e Amizade entre a Repúbica Federativa do Brasil e o Reino da Espanha (1994). Decreto $\mathrm{n}^{\circ}$ 1.211. // Presidência da República. (1994). http://www.planalto.gov.br/ccivil_03/decreto/19901994/D1211.htm (2016-06-16).

Enviado: 2016-08-16. Segunda versión: 2017-02-22. Aceptado: 2017-05-09. 